\title{
Existence of Equilibrium in Common Agency Games with Adverse Selection*
}

\author{
Guilherme Carmona $^{\dagger}$ \\ José Fajardo \\ Universidade Nova de Lisboa IBMEC Business School
}

July 11, 2006

\begin{abstract}
We establish the existence of sequential equilibria in general menu games, known to be sufficient to analyze common agency problems. In particular, we show that our result solves some unpleasant features of early approaches.
\end{abstract}

Keywords: Common Agency, Menu Games, Sequential Equilibrium.

*We wish to thank Luís Vasconcelos and Mehmet Barlo for very helpful comments. We thank also John Huffstot for editorial assistance. Any remaining error is, of course, our own.

${ }^{\dagger}$ Address: Universidade Nova de Lisboa, Faculdade de Economia, Campus de Campolide, 1099-032 Lisboa, Portugal; Phone: (351) 21380 1671; Fax: (351) 21387 0933; email: gcarmona@fe.unl.pt.

¥Address: IBMEC Business School, Av. Rio Branco 108, 20040 001, Rio de Janeiro, Brazil; Phone: (55) 214503 4162; Fax: (55) 214503 4168; email: pepe@ibmecrj.br. 


\section{Introduction}

In many important examples in multi-contracting mechanism design, several principals (attempt to) contract with a common agent to influence her choice. Such a common agency model has been the focus of much of the recent research in incentive theory. ${ }^{1}$

In the common agency model, principals offer a menu of contracts to the agent, who chooses one contract from the ones being offered. Although one could imagine more general communication channels between the principals and the agent, Martimort and Stole (2002), Page and Monteiro (2003), Peters (2001) and Peters (2003) have shown that such a procedure of offering menus of contracts is enough to characterize the set of equilibrium allocations. In fact, as Martimort (2006) points out, "what matters per se is not the kind of communication that a principal uses with his agent but the set of options that this principal makes available to the agent." This result, known as the delegation principle, implies that the common agency problem can be analyzed through a menu game.

However, in order for the delegation principle to be meaningful, an equilibrium must exist. In this paper, we present a solution to this problem by establishing a general existence theorem for menu games.

In our approach, a menu game is modeled through a game with incomplete information. In its extensive form, Nature moves first, choosing the agent's type. Following this choice, the first principal chooses a menu of contracts (defined as a closed subset of the contract space) without observing the agent's type. Then, the second principal offers his menu of contracts, ob-

\footnotetext{
${ }^{1}$ See Martimort (2006) for a survey.
} 
serving neither the agent's type nor the menu offered by the first principal. After all principals have made their offers, the agent chooses one contract (or one contract of each principal), knowing her type and the menus offered by the principals.

Since a menu game is a game with incomplete information, we focus on sequential equilibria. As we allow for a Polish space of types and a compact metric space of contracts, its extensive form will, typically, be infinite. Despite this technical difficulty, it is easy to define the notion of sequential equilibrium of a menu game. In fact, each principal has a unique information set and all of the agent's information sets are singletons. Therefore, an assessment, i.e. a pair of beliefs and strategies, is consistent if beliefs are defined using the strategy and Bayes' rule. Moreover, a consistent assessment is sequentially rational if: (1) the agent optimizes at every possible type and vector of menus and (2) each principal optimizes given the strategy of the other principals and the strategy of the agent. ${ }^{2}$

We can rephrase the above description of sequential equilibria in menu games in the following way. Note that a strategy for the agent induces a normal-form game between the principals. In fact, this is a game where each principal has the set of all possible menus as his own pure strategy set and his payoff is determined by the choice of all principals together with the agent's strategy. Thus, a sequential equilibrium consists of the beliefs described above, an optimal strategy for the agent and a Nash equilibrium for the normal-form game induced by such strategy.

\footnotetext{
${ }^{2}$ Thus, the set of sequential equilibrium strategies coincides with the set of perfect Bayesian equilibria.
} 
The above description of a sequential equilibrium is useful because we can regard the family of normal-form games induced by the agent's strategies as a game with an endogenous sharing rule as in Simon and Zame (1990) and, therefore, use their existence theorem to establish the existence of sequential equilibria in menu games. In fact, a vector of menus defines a subset of payoffs for the principals, each of which corresponds to a particular strategy of the agent. This clearly defines a correspondence from principals' strategies into payoffs as required for a game with an endogenous sharing rule.

In order to use Simon and Zame's theorem in our setting, we need to generalize it along two dimensions. The first is relatively straightforward and amounts to allowing the payoff correspondence to depend on the agent's type. Using a similar approach as in Simon and Zame (1990), we show that any such generalized game with endogenous sharing rules has a solution provided that, on top of their assumptions, the payoff correspondence is measurable.

A second generalization is needed in order to obtain a sequential equilibrium from a solution. A solution for a (generalized) game with an endogenous sharing rule is a strategy for the principals and a measurable selection from the payoff correspondence such that the strategy is a Nash equilibrium of the normal-form game with that selection as its payoff function. Hence, an answer to our problem can be obtained by addressing the following question: is there a measurable selection from the agent's optimal choice correspondence such that, when composed with the principals' payoff function, equals the selection from the payoff correspondence which is part of a solution? Our second generalization addresses this question, showing that when the payoff correspondence is the composition of players' payoff functions with some 
correspondence (interpreted as the optimal choice correspondence of players whose behavior is not explicitly modeled), then every measurable selection from the payoff correspondence can be obtained as the composition of players' payoff function and a measurable selection of this other correspondence. Combining our two generalizations, we show that every menu game satisfying enough continuity properties has a sequential equilibrium.

The existence of equilibrium in menu games has also been addressed by Page and Monteiro (2003) and Monteiro and Page (2005). The main difference between our approach and theirs is that they focus on a normal-form game played by the principals. In Monteiro and Page (2005), they fixed exogenously an optimal strategy for the agent, and then proceed to address the existence of a Nash equilibrium in the resulting normal-form game. A similar approach is used in Page and Monteiro (2003), although there the payoff function used by the principals is defined differently and cannot, in general, be induced by an optimal strategy of the agent. Due to those differences, we were able to find examples of equilibria in their sense there are not part of any sequential equilibrium, and also, examples of sequential equilibrium strategies for the principals that are not equilibria in their sense (see Carmona and Fajardo (2006)).

Nevertheless, in menu games with exclusivity (i.e., in which the agent chooses only one principal) and no fixed costs (i.e., a principal receives a zero payoff if not chosen by the agent), we show in Carmona and Fajardo (2006) that every equilibrium in the sense of Monteiro and Page (2005) is part of a sequential equilibrium. Thus, for this class of games, their main result provides a solution to the existence question that has the advantage of 
allowing for principals' payoff functions that are only upper semicontinuous and integrably bounded and not continuous and bounded, as required by our existence result. In contrast, our existence result can be applied in general menu games, requiring neither the exclusivity and no-fixed-cost assumptions nor the particular payoff functions for both the agent and the principals used by Monteiro and Page (2005).

The paper is organized as follows. In Section 2 we present the model. Then, we present the definition and characterization of sequential equilibria in Section 3. We establish our existence result in Section 4. Section 5 concludes.

\section{Menu Games}

Consider a game with $m$ principals who can offer contracts to a single agent. The set of contracts that principal $i$ can offer is denoted by $K_{i}$ and we assume that $K_{i}$ is a compact metric space. Each principal offers a menu of contracts to the agent. A menu of contracts for principal $i \in I=\{1, \ldots, m\}$ is just a nonempty closed subset $C_{i}$ of $K_{i}$.

In Martimort and Stole (2002) for example, the set of contracts that a principal can offer equals the set of probability measures over the allocations controlled by him. Under the assumption that the set of these allocations is finite, it follows that each principal's contract space is compact. They allow each principal $i$ to offer a mechanism to the agent, consisting of a message space $\mathcal{M}_{i}$ and an outcome function $g_{i}: \mathcal{M}_{i} \rightarrow K_{i}$. If $\mathcal{M}_{i}$ is compact and $g_{i}$ is continuous, then the menu $g_{i}\left(\mathcal{M}_{i}\right)$ induced by $\left(\mathcal{M}_{i}, g_{i}\right)$ is a closed subset 
of $K_{i}$. Thus, in this setting, assuming that menus are closed subsets of $K_{i}$ amounts to assuming that principals use mechanisms with compact message spaces and continuous outcome functions.

Let $P_{i}$ be the collection of all nonempty, closed subsets of $K_{i}$. It is well known that $P_{i}$ is a compact metric space when endowed with the Hausdorff metric. Let $P=P_{1} \times \cdots \times P_{m}$ and $C=\left(C_{1}, \ldots, C_{m}\right)$ denote a profile of menus.

Let $\mathbb{K}$ denote the pure action space of the agent. We assume that $\mathbb{K}$ is a compact metric space and we let $k$ denote a generic element of $\mathbb{K}$. There are two particular cases for $\mathbb{K}$ in which we are interested. One, considered in Page and Monteiro (2003), is $\mathbb{K}^{P M}=\left\{(i, f) \in I \times \cup_{i=1}^{m} K_{i}: f \in K_{i}\right\}$, where $I=\{1, \ldots, m\}$. Here, the agent chooses the principal with whom she wishes to contract and chooses one contract from this principal. Implicitly, the assumption is that contracts are exclusive.

A second particular case, considered in Martimort and Stole (2002), is $\mathbb{K}^{M S}=K_{1} \times \cdots \times K_{m}$. In this case, contracts are not exclusive and so the agent can choose a contract from each principal.

These two cases can be combined in a hybrid model in which a subset $I_{e} \subseteq I$ of principals only allows for exclusive contracts. In this case, let $\mathbb{K}^{H}=\left\{(i, f) \in I_{e} \times \cup_{i \in I_{e}} K_{i}: f \in K_{i}\right\} \times \prod_{i \in I_{e}^{c}} K_{i} \cdot{ }^{3}$ It is clear that $\mathbb{K}^{P M}$, $\mathbb{K}^{M S}$ and $\mathbb{K}^{H}$ are compact.

The agent's payoff depends on her type, which is described by an element of a Polish space $T$ (i.e., $T$ is a complete separable metric space). We endow

\footnotetext{
${ }^{3}$ In all the above models, we can let some $f \in K_{i}$ denote no contracting, following Page and Monteiro (2003).
} 
$T$ with its Borel $\sigma$ - algebra and let $\mu$ describe the probability measure on the set of types. ${ }^{4}$ The agent's utility function is $v: T \times \mathbb{K} \rightarrow \mathbb{R}$ and we assume that $v$ is a Carathéodory function. ${ }^{5}$

The agent's problem is as follows. Knowing $t \in T$ and given a menu profile $C$ offered by the principals, she can choose a mixed strategy over $\mathbb{K}$. A mixed strategy is a Borel probability measure on $\mathbb{K}$ and we let $\Delta(\mathbb{K})$ denote the space of all such probability measures. The set of available mixed strategies is described by a nonempty compact convex set $\varphi(t, C) \subseteq \Delta(\mathbb{K})$. Furthermore, we assume that the correspondence $\varphi: T \times P \rightrightarrows \Delta(\mathbb{K})$ is continuous.

The idea behind the constraint correspondence $\varphi$ is that the agent can choose only among the contracts being offered, i.e., she can only choose a contract $f_{i} \in C_{i}$ from principal $i$. Therefore, we have three possible specifications for $\varphi$ corresponding to the above particular cases for $\mathbb{K}$ :

$$
\varphi^{P M}(t, C)=\left\{\lambda \in \Delta\left(\mathbb{K}^{P M}\right): \lambda\left(\cup_{i=1}^{m}\left(\{i\} \times C_{i}\right)\right)=1\right\}
$$

in the exclusivity case,

$$
\varphi^{M S}(t, C)=\left\{\lambda \in \Delta\left(\mathbb{K}^{M S}\right): \lambda(C)=1\right\}
$$

in the non-exclusivity case and

$$
\varphi^{H}(t, C)=\left\{\lambda \in \Delta\left(\mathbb{K}^{H}\right): \lambda\left(\cup_{i \in I_{e}}\left(\{i\} \times C_{i}\right) \times \prod_{i \in I_{e}^{c}} C_{i}\right)=1\right\}
$$

\footnotetext{
${ }^{4}$ Throughout the paper, we endow all metric spaces we consider with their Borel $\sigma-$ algebra. Therefore, we abbreviate Borel-measurable by measurable.

${ }^{5}$ If $(S, \Sigma)$ is a measurable space, $X$ and $Y$ are topological spaces and $f: S \times X \rightarrow Y$ is a function, then $f$ is a Carathéodory function if it is measurable in $s$ and continuous in $x$.
} 
in the hybrid case. Lemma 7 in Appendix shows that $\varphi^{P M}, \varphi^{M P}$ and $\varphi^{H}$ are continuous with nonempty, convex and compact values.

Hence, given $t \in T$ and $C \in P$, the agent's problem is

$$
\max _{\lambda \in \varphi(t, C)} \int_{\mathbb{K}} v(t, k) \mathrm{d} \lambda(k) .
$$

Let $\Lambda: T \times P \rightrightarrows \Delta(\mathbb{K})$ denote the correspondence of optimal choices. A strategy for the agent is then a measurable function $\sigma: T \times P \rightarrow \Delta(\mathbb{K})$, and, clearly, $\sigma$ is an optimal strategy if and only if it is a selection of $\Lambda$.

We now turn to the principals' problem. Principals choose simultaneously. For all $i \in I$, principal $i$ 's choice set is $\Delta\left(P_{i}\right)$, the set of mixed strategies on $P_{i}$ and his profit function is denoted by $\pi_{i}: T \times \mathbb{K} \rightarrow \mathbb{R}$. We assume that $\pi_{i}$ is a bounded Carathéodory function. ${ }^{6}$

If the principals offer a menu $C=\left(C_{1}, \ldots, C_{m}\right) \in P$ and the agent uses a strategy $\sigma: T \times P \rightarrow \Delta(\mathbb{K})$, then principal $i$ 's payoff is

$$
F_{i}(t, C ; \sigma)=\int_{\mathbb{K}} \pi_{i}(t, k) \mathrm{d} \sigma(k \mid t, C) .
$$

Since $\sigma$ is measurable, then so is the real-valued function $F_{i}$ on $T \times P$. Finally, if principals choose strategies $\alpha=\left(\alpha_{1}, \ldots, \alpha_{m}\right)$ and the agent chooses a strategy $\sigma$,

$$
F_{i}(\alpha ; \sigma)=\int_{P} \int_{T} F_{i}(t, C ; \sigma) \mathrm{d} \mu(t) \mathrm{d} \alpha(C)
$$

denotes principal $i$ 's payoff.

A menu game is denoted by $G$ and we use $G^{P M}, G^{M S}$ and $G^{H}$ to denote particular menu games for the corresponding choices of $\mathbb{K}$ and $\varphi$ mentioned

\footnotetext{
${ }^{6}$ Note that we are strengthening the assumptions made by Page and Monteiro (2003) since they have only assumed that $t \mapsto \pi_{i}(t, k)$ is measurable, $k \mapsto \pi_{i}(t, k)$ is upper semicontinuous and that $\pi_{i}$ is $\mu$-integrably bounded.
} 
above. We say that a menu game $G$ is continuous if it satisfies all the above assumptions.

\section{Sequential Equilibrium}

We can think of a menu game as being played in the following way. First, nature chooses a type for the agent and then, without knowing this, principals simultaneously choose a menu. Finally, the agent knowing her type and the menu choices of every principal, chooses an element of $\mathbb{K}$.

We can use an extensive form to describe a menu game $G$. Each initial note corresponds to a choice of a type for the agent by nature, and so, we index those nodes by $t \in T$. Principal 1 is the first to choose. Since he does not observe the type of the agent, principal 1's information set $H_{1}$ consists of the set of initial nodes; thus, slightly abusing notation, we write $H_{1}=T$. At $H_{1}$, principal 1 chooses a menu $C_{1} \in P_{1}$, which is not observed by principal 2. Therefore, principal 2's information set $H_{2}$ is equal to $\left\{\left(t, C_{1}\right): t \in\right.$ $T$ and $\left.C_{1} \in P_{1}\right\}=T \times P_{1}$. Proceeding in this way, let $H_{i}=T \times P_{1} \times \cdots \times P_{i-1}$. Finally, the agent observes her type and the choice of each principal and so the family of her (singleton) information sets is $\{(t, C): t \in T$ and $C \in P\}$. She chooses an element $k \in \mathbb{K}$ and a terminal node is reached. Payoffs are then determined using the agent's and principals' payoff functions.

Since we represent a menu game by a game with incomplete information, it is natural to use sequential equilibrium as the solution concept (see Kreps and Wilson (1982)). Recall that an assessment is a pair of beliefs $\nu$ and strategies $(\alpha, \sigma)$ and that a sequential equilibrium is a consistent sequen- 
tially rational assessment. Since there are (possibly) an infinite number of information sets, we must explicitly require that strategies - functions from information sets into actions - be measurable.

Consistent assessments $(\nu,(\alpha, \sigma))$ are easy to characterize in a menu game. First, since each of the agent's information sets is a singleton, her belief there must assign probability one to that single point. Second, if $\nu_{i}$ denotes the belief in principal $i$ 's information set $H_{i}$, and $B$ is a measurable subset of $H_{i}$, then $\nu_{i}(B)=\mu \times \alpha_{1} \times \cdots \times \alpha_{i-1}(B)$. This follows because if the probability distribution induced by $(\alpha, \sigma)$ on the nodes of the extensive form is denoted by $P^{\alpha, \sigma}$, then $P^{\alpha, \sigma}\left(H_{i}\right)=1$ and so

$$
\nu_{i}(B)=P^{\alpha, \sigma}\left(B \mid H_{i}\right)=P^{\alpha, \sigma}(B)=\mu \times \alpha_{1} \times \cdots \times \alpha_{i-1}(B) .
$$

Using these beliefs, sequential equilibria of menu games are characterized in the following remark.

Remark 1 An assessment $(\nu,(\alpha, \sigma))$ is a sequential equilibrium of a menu game $G$ if and only if

1. $\nu_{i}=\mu \times \alpha_{1} \times \cdots \times \alpha_{i-1}$ for all $i \in I$,

2. $\sigma$ is a measurable selection of $\Lambda$ and

3. $F_{i}(\alpha ; \sigma) \geq F_{i}\left(\bar{\alpha}_{i}, \alpha_{-i} ; \sigma\right)$ for all $i \in I$ and $\bar{\alpha}_{i} \in \Delta\left(P_{i}\right)$.

Thus, in a sequential equilibrium of $G$, beliefs are determined by Bayes' rule, the agent optimizes for all possible types and menus offered, and each principal optimizes given the strategy of the other principals and the strategy of the agent. 


\section{Existence of Sequential Equilibrium}

Our main result is the following existence theorem.

Theorem 1 A sequential equilibrium exists for all continuous games $G$.

Since the frameworks of Page and Monteiro (2003) and Martimort and Stole (2002) are particular cases of ours, we have the following corollary.

Corollary 1 All continuous games $G^{H}, G^{P M}$ and $G^{M S}$ have a sequential equilibrium.

In order to establish Theorem 1 we first generalize the theorem in Simon and Zame (1990) by allowing the payoff correspondence to depend on the agent's type. We then use this result to prove the existence of a sequential equilibrium in any continuous menu game. This last argument uses Lemma 2 , below, which shows how to obtain a sequential equilibrium from a solution of a (generalized) game with an endogenous sharing rule.

\subsection{A Generalization of Simon and Zame's Theorem}

A generalized game with an endogenous sharing rule is $G=\left(P_{1}, \ldots, P_{m}, T, Q\right)$ satisfying $P_{i}$ is a compact metric space for all $i, T$ is a Polish space and $Q: T \times P \rightrightarrows \mathbb{R}^{m}$ is measurable, bounded, has nonempty, convex and compact values and is upper hemi-continuous in $C$ for all $t \in T$ (i.e., $C \mapsto Q(t, C)$ is upper hemi-continuous for all $t \in T$ ).

A solution for $G$ is a pair $(q, \alpha)$ such that $q$ is a measurable selection of $Q, \alpha_{i} \in \Delta\left(P_{i}\right)$ and

$$
\int_{P} \int_{T} q_{i}(t, C) \mathrm{d} \mu(t) \mathrm{d} \alpha(C) \geq \int_{P} \int_{T} q_{i}(t, C) \mathrm{d} \mu(t) \mathrm{d}\left(\beta_{i} \times \alpha_{-i}\right)(C)
$$


for all $i$ and all $\beta_{i} \in \Delta\left(P_{i}\right)$.

Theorem 2 A solution exists for all generalized games with an endogenous sharing rule.

The proof of Theorem 2 follows closely the one in Simon and Zame (1990) and is presented in Appendix A.1.

\subsection{Proof of Theorem 1}

The proof of Theorem 1 proceeds as follows: first, we define a generalized game with an endogenous sharing rule, essentially, defining the payoff correspondence by composing principals' payoff functions with the optimal choice correspondence of the agent. We then use Theorem 2 to obtain a solution to that generalized game with an endogenous sharing rule. Then, we use Lemma 2 to show that the measurable selection from the payoff correspondence can be written as the composition between the principals' payoff function and a measurable selection from the agent's optimal choice correspondence (i.e., an optimal strategy for the agent). Finally, we show that this strategy together with the principals' strategies that are part of the solution for the generalized game with an endogenous sharing rule form a sequential equilibrium strategy.

Let $h: T \times P \times \Delta(\mathbb{K}) \rightarrow \mathbb{R}^{m}$ be defined by

$$
h(t, C, \lambda)=\int_{\mathbb{K}} \pi(t, k) \mathrm{d} \lambda(k) .
$$

Note that if $\sigma: T \times P \rightarrow \Delta(\mathbb{K})$ is a strategy for the agent, then $F(t, C ; \sigma)=$ $h(t, C, \sigma(t, C))$ for all $t \in T$ and $C \in P$. Also, note that $h$ is independent of 
$C, \lambda \mapsto h(t, C, \lambda)$ is continuous and $(t, C) \mapsto h(t, C, \lambda)$ is measurable. Hence, $h$ is a Carathéodory function. Define $Q: T \times P \rightrightarrows \mathbb{R}^{m}$ by

$$
Q(t, C)=\{h(t, C, \lambda): \lambda \in \Lambda(t, C)\}
$$

Lemma 1 The correspondence $\Lambda$ is measurable and has compact values. The correspondence $Q$ is measurable, bounded, upper hemi-continuous in $C$ for all $t \in T$ and has nonempty, convex and compact values.

It follows by Lemma 1 that $G=\left(P_{1}, \ldots, P_{m}, T, Q\right)$ is a generalized game with an endogenous sharing rule. Hence, by Theorem 2, there exists a solution $(q, \alpha)$ for $G$.

In order to obtain a sequential equilibrium from the solution $(q, \alpha)$, we use the following lemma.

Lemma 2 Let $S$ be a measurable space, $X$ be a compact metric space, $g$ : $S \times X \rightarrow \mathbb{R}^{m}$ a Carathéodory function and $\Theta: S \rightrightarrows X$ a compact valued, measurable correspondence.

If $Q: S \rightrightarrows \mathbb{R}^{m}$ is defined by

$$
Q(s)=\{g(s, x): x \in \Theta(s)\}
$$

for all $s \in S$ and $q$ is a measurable selection of $Q$, then there exists a measurable selection $\alpha$ of $\Theta$ such that $q(s)=g(\alpha(s))$ for all $s \in S$.

Since $T \times P$ is a measurable space, $\Delta(\mathbb{K})$ is a compact metric space and $\Lambda$ is compact valued and measurable, then by Lemma 2, there exists a measurable selection $\sigma$ from $\Lambda$ such that $q(t, C)=h(t, C, \sigma(t, C))$ for all 
$t \in T$ and $C \in P$. Hence,

$$
\begin{aligned}
& F_{i}(\alpha ; \sigma)=\int_{P} \int_{T} h_{i}(t, C, \sigma(t, C)) \mathrm{d} \mu(t) \mathrm{d} \alpha(C)= \\
& \int_{P} \int_{T} q_{i}(t, C) \mathrm{d} \mu(t) \mathrm{d} \alpha(C) \geq \int_{P} \int_{T} q_{i}(t, C) \mathrm{d} \mu(t) \mathrm{d}\left(\beta_{i} \times \alpha_{-i}\right)(C)= \\
& \int_{P} \int_{T} h_{i}(t, C, \sigma(t, C)) \mathrm{d} \mu(t) \mathrm{d}\left(\beta_{i} \times \alpha_{-i}\right)(C)=F_{i}\left(\beta_{i}, \alpha_{-i} ; \sigma\right)
\end{aligned}
$$

for all $i$ and all $\beta_{i} \in \Delta\left(P_{i}\right)$. It then follows that, if $\nu$ is as in Remark 1, then $(\nu,(\alpha, \sigma))$ is a sequential equilibrium of $G$.

\section{Conclusions}

We have shown that a sequential equilibrium exists in all continuous menu games. Compared with the results of Page and Monteiro (2003) and Monteiro and Page (2005), our existence theorem has the advantage of dispensing with the exclusivity and the no-fixed-cost assumptions made in those papers.

Our approach also has the advantage of being simpler than the one used by Monteiro and Page (2005). In fact, they choose an optimal strategy for the agent and then proceed by studying the challenging problem of the existence of a Nash equilibrium for the resulting normal-form game played by the principals. In contrast, we proceed by determining the agent's strategy endogenously, which, as Simon and Zame (1990) have pointed out, simplifies the existence problem considerably.

Our approach relies heavily on the ideas of Simon and Zame (1990). In fact, the proof of our existence result is straightforward once we extend their theorem, first, to the case in which the principals' payoff correspondence depends on the agent's type and, second, to the case where it equals the 
composition of the agent's optimal choice correspondence and principals' payoff functions. This second result allows us to obtain an equilibrium in the usual sense from a solution in the sense of Simon and Zame (1990).

We hope that these two results prove to be useful in analyzing similar problems, for instance, the principal-agent relationship with an informed principal of Maskin and Tirole (1990) and its generalization to a common agency problem considered in Martimort and Moreira (2006).

\section{A Appendix}

In the appendix, we prove Theorem 2, and Lemmas 1 and 2. Also, we prove that the correspondences $\varphi^{P M}, \varphi^{M S}$ and $\varphi^{H}$ are continuous with nonempty, convex, compact values.

\section{A.1 Proof of Theorem 2}

Our proof of Theorem 2 follows the one in Simon and Zame (1990). Indeed, we start by modifying their Lemma 2 and then proceed by adapting the six steps of their proof to our setting.

Both their Lemma 2 and our version of it applies to vector-valued measures defined as follows. If $S$ is a Polish space, $q$ is a bounded, measurable function from $S$ into $\mathbb{R}^{m}$ and $\psi$ is a probability measure on $S$, define $q \psi \in \Delta(S)$ by

$$
q \psi(B)=\int_{B} q \mathrm{~d} \psi
$$

for all measurable subsets $B$ of $S$. The following two lemmas consider the special case where $S=T \times P$ and $\psi=\mu \times \alpha$ for some $\alpha \in \Delta(P)$. They 
establish some properties of $q(\mu \times \alpha)$ that are useful in our version of Simon and Zame's Lemma 2.

Lemma 3 If $\alpha \in \Delta(P)$ and $q: T \times P \rightarrow \mathbb{R}^{m}$ is bounded and measurable, then

$$
q(\mu \times \alpha)=\mu \times q \alpha .
$$

Proof. Let $A$ and $B$ be measurable subsets of $T$ and $P$, respectively. Then,

$$
\begin{aligned}
q(\mu \times \alpha)(A \times B) & =\int_{A}\left(\int_{B} q \mathrm{~d} \alpha\right) \mathrm{d} \mu \\
& =\int_{A} q \alpha(B) \mathrm{d} \mu \\
& =\mu(A) q \alpha(B) .
\end{aligned}
$$

Hence, the result follows from Hildenbrand (1974, Theorem 24, p. 47).

Let $q: T \times P \rightarrow \mathbb{R}^{m}$ be bounded and measurable and define $\hat{q}: P \rightarrow \mathbb{R}^{m}$ by

$$
\hat{q}(C)=\int_{T} q(t, C) \mathrm{d} \mu(t)
$$

If $X$ and $Y$ are metric spaces and $\nu$ is a measure on $X \times Y, \nu_{Y}$ denotes the marginal distribution of $\nu$ on $Y$.

Lemma 4 If $q: T \times P \rightarrow \mathbb{R}^{m}$ is bounded and measurable, then $\hat{q}$ is measurable and

$$
\hat{q} \alpha=q(\mu \times \alpha)_{P}
$$

Proof. Since $q$ is bounded and measurable, the integral exists. The measurability of $\hat{q}$ follows from Fubini's Theorem (see Aliprantis and Border (1999, Theorem 11.26, p. 411)). 
We turn to the second claim. Let $B$ be a measurable subset of $P$. It follows that

$$
\begin{aligned}
& \hat{q} \alpha(B)=\int_{B} \hat{q} \mathrm{~d} \alpha=\int_{B} \int_{T} q \mathrm{~d} \mu \mathrm{d} \alpha= \\
& \int_{T \times B} q \mathrm{~d}(\mu \times \alpha)=q(\mu \times \alpha)(T \times B)=q(\mu \times \alpha)_{P}(B) .
\end{aligned}
$$

Thus, the lemma follows.

After these preliminaries, we turn to our version of Lemma 2 in Simon and Zame (1990). There, we allow for the case where $S$ is the product of a Polish space (i.e., a complete separable metric space) and a compact metric space and $Q$ is measurable but only upper hemi-continuous in the second variable. However, we assume that all the measures involved are finite.

Lemma 5 Let $\left\{\nu_{n}\right\}$ be a sequence of probability measures on $P$ converging weakly to $\nu$ and let $Q: T \times P \rightrightarrows \mathbb{R}^{m}$ be a bounded, measurable correspondence, upper hemi-continuous in $C$ for all $t \in T$ and with compact, convex, nonempty values. For each $n$, let $q_{n}$ be a measurable selection from $Q$.

If the sequence $\left\{q_{n}\left(\mu \times \nu_{n}\right)\right\}$ of vector-valued measures converges weakly to a vector-valued measure $\xi$, then there exists a measurable selection $q$ from $Q$ such that $\xi=q(\mu \times \nu)$.

Proof. Note that $\left\{\mu \times \nu_{n}\right\}$ converges weakly to $\{\mu \times \nu\}$ by Hildenbrand (1974, Theorem 27, pg. 49). Therefore, the boundedness of $Q$ implies, as in Simon and Zame (1990, Lemma 2), that there exists a measurable function $q: T \times P \rightarrow \mathbb{R}^{m}$ such that $\xi=q(\mu \times \nu)$.

Let $H=\{(t, C) \in T \times P: q(t, C) \notin Q(t, C)\}$. Since both $q$ and $Q$ are measurable, then $H$ is measurable. In fact, let $S=T \times P$ for convenience, 
$f=\left(i d_{S}, q\right)$ and $\delta: S \times \mathbb{R}^{m} \rightarrow \mathbb{R}$ be defined by $\delta(s, x)=\max _{z \in Q(s)}\|x-z\|$. Clearly, $f$ is measurable since $q$ is also measurable. Since $Q$ is measurable, then $Q$ is weakly measurable by Theorem 17.2 in Aliprantis and Border (1999, p. 559). Then, $\delta$ is a Carathéodory function by Theorem 17.5 in Aliprantis and Border (1999, p. 562) and thus measurable since $\mathbb{R}^{m}$ is separable (see Aliprantis and Border (1999, Lemma 4.50, p.151)). It follows that the function $g: S \rightarrow \mathbb{R}$ defined by $g=\delta \circ f$ is measurable and that $H=\{s \in S: g(s)>0\}$ is a measurable subset of $S$.

Let $t \in T$ and $H_{t}=\{C \in P:(t, C) \in H\}$. Since $H$ is a measurable subset of $T \times P$, then $H_{t}$ is a measurable subset of $P$ (see Aliprantis and Border (1999, Lemma 4.45, p. 148)). Since $P$ is compact and $c \mapsto Q(t, c)$ is upper hemi-continuous, it follows by the arguments of Simon and Zame that $\nu\left(H_{t}\right)=0$. Thus,

$$
\mu \times \nu(H)=\int_{T} \nu\left(H_{t}\right) \mathrm{d} \mu(t)=0
$$

by Fubini's Theorem. This completes the proof since we can correct $q$ in $H$, obtaining a function that is still measurable.

We turn to the proof of Theorem 2, showing that the same arguments used by Simon and Zame extend to our setting, with minor changes.

Step 1: Finite approximations. Recall that $P$ is a compact metric space. As in Simon and Zame (1990), discretize $P$ in order to obtain, for all $r \in \mathbb{N}$, a finite action space $P_{i}^{r}$ for all players $i=1, \ldots, m$, a measurable selection $q^{r}$ from $Q$ and a solution $\left(\alpha_{1}^{r}, \ldots, \alpha_{m}^{r}\right)$ for $G^{r}=\left(P_{1}^{r}, \ldots, P_{m}^{r}, T, Q\right)$. Let $\alpha^{r}=\alpha_{1}^{r} \times \cdots \times \alpha_{m}^{r}$.

Step 2: Limits. Since $\Delta\left(P_{i}\right)$ is compact, then we may assume that $\left\{\alpha_{i}^{r}\right\}$ 
converges for all $i=1, \ldots, m$. Thus, $\left\{\alpha^{r}\right\}$ converges. Letting $\alpha=\lim _{r} \alpha^{r}$, it follows that $\left\{\mu \times \alpha^{r}\right\}$ converges to $\mu \times \alpha$.

Similarly, we may assume that $\left\{q^{r} \alpha^{r}\right\}$ converges. This, together with Lemma 3, implies that $\left\{q^{r}\left(\mu \times \alpha^{r}\right)\right\}=\left\{\mu \times q^{r} \alpha^{r}\right\}$ converges. Let $\xi=$ $\lim _{r} q^{r}\left(\mu \times \alpha^{r}\right)$.

Step 3: Selections. By Lemma 5, there exists a measurable selection $q$ from $Q$ such that $\xi=q(\mu \times \alpha)$.

Step 4: Better responses. Recall that $\hat{g}: P \rightarrow \mathbb{R}^{m}$ is defined by $\hat{g}(C)=\int_{T} g(t, C) \mathrm{d} \mu(t)$ for all bounded and measurable functions $g: T \times P \rightarrow$ $\mathbb{R}^{m}$.

By Lemma 4, $\hat{q}$ is measurable and $\alpha_{r}$ is a Nash equilibrium of the normalform game $\left(P_{1}^{r}, \ldots, P_{m}^{r}, \hat{q}\right)$ for all $r \in \mathbb{N}$. Since $\hat{q} \alpha=q(\mu \times \alpha)_{P}$ and, similarly, $\hat{q}^{r} \alpha^{r}=q^{r}\left(\mu \times \alpha^{r}\right)_{P}$, it follows that $\left\{\hat{q}^{r} \alpha^{r}\right\}$ converges to $\hat{q} \alpha$.

If $X$ is a metric space and $x \in X$, let $\delta_{x}$ denote the probability measure on $X$ degenerate on $x$. Letting

$$
H_{i}=\left\{C_{i} \in P_{i}: \int_{P} \int_{T} q_{i} \mathrm{~d} \mu \mathrm{d}\left(\delta_{C_{i}} \times \alpha_{-i}\right)>\int_{P} \int_{T} q_{i} \mathrm{~d} \mu \mathrm{d}\left(\alpha_{i} \times \alpha_{-i}\right)\right\},
$$

it follows from Simon and Zame (1990, Step 4) that

$$
\alpha_{i}\left(H_{i}\right)=\alpha_{i}\left(\left\{x_{i} \in P_{i}: \int_{P} \hat{q}_{i} \mathrm{~d}\left(\delta_{C_{i}} \times \alpha_{-i}\right)>\int_{P} \hat{q}_{i} \mathrm{~d}\left(\alpha_{i} \times \alpha_{-i}\right)\right\}\right)=0 .
$$

Step 5: Perturbation. As in Step 5 of Simon and Zame (1990), for all $i$, let $p^{i}: T \times P \rightarrow \mathbb{R}^{m}$ be any measurable selection from $Q$ which minimizes 
the $i$ th component. Let $Y=\left\{C \in P: C_{i} \in H_{i}\right.$ for at least two indices $\left.i\right\}$ and define $f: T \times P \rightarrow \mathbb{R}^{m}$ as follows:

$$
f(t, C)= \begin{cases}p^{i}(t, C) & \text { if } C \in H_{i} \times P_{-i} \text { but } C \notin Y, \\ q(t, C) & \text { otherwise. }\end{cases}
$$

Since $\alpha\left(H_{i} \times P_{-i}\right)=0$ for all $i \in I$, then

$$
\int_{P} \int_{T} f \mathrm{~d} \mu \mathrm{d} \alpha=\int_{P \backslash \cup_{i=1}^{m}\left(H_{i} \times P_{-i}\right)} \int_{T} q \mathrm{~d} \mu \mathrm{d} \alpha=\int_{P} \int_{T} q \mathrm{~d} \mu \mathrm{d} \alpha .
$$

Therefore,

$$
\int_{P} \hat{f} \mathrm{~d} \alpha=\int_{P} \hat{q} \mathrm{~d} \alpha
$$

Let $i \in I$. If $x_{i} \notin H_{i}$, then

$$
\hat{q}_{i}(C)=\hat{f}_{i}(C)
$$

except possibly for $C \in\left[\left\{C_{i}\right\} \times P_{-i}\right] \cap\left[\cup_{j \neq i}\left(H_{j} \times P_{-j}\right)\right]$. Finally, we also have that

$$
\hat{p}_{i}^{i}(C)=\hat{f}_{i}(C)
$$

for all $C \in H_{i} \times P_{-i}$ and $C \notin Y$.

Step 6: Solution. Note that $p_{i}^{i}: T \times P \rightarrow \mathbb{R}$ is lower semi-continuous in $C$ for all $t \in T$, as in Simon and Zame (1990, step 6). Thus, it follows from Fatou's Lemma (see Aliprantis and Border (1999, Theorem 11.19, p. 407)) that $\hat{p}_{i}^{i}: P \rightarrow \mathbb{R}$ is lower semi-continuous. Because of equations (10), (11) and (12), it follows from Simon and Zame (1990, Step 6) that $\alpha$ is a Nash equilibrium of the normal-form game $\left(P_{1}, \ldots, P_{m}, \hat{f}\right)$. Since $\hat{f}=\int_{T} f \mathrm{~d} \mu$, it follows that $(f, \alpha)$ is a solution of $G=\left(P_{1}, \ldots, P_{m}, T, Q\right)$.

This completes the proof of Theorem 2 . 


\section{A.2 Proof of Lemma 1}

For convenience, let $S=T \times P$. Note first that $\Lambda$ is measurable by Theorem 17.18 in Aliprantis and Border (1999, p. 570) and has nonempty, convex and compact values. Furthermore, by Berge's Maximum Theorem (see Berge (1997, p. 116)), the correspondence $C \mapsto \Lambda(t, C)$ is upper hemi-continuous for all $t \in T$.

Then, $Q$ is bounded since $\pi$ is bounded and $Q$ is nonempty valued since $\Lambda$ is also nonempty valued. Since for all $s \in S, \Lambda(s)$ is compact, $Q(s)=$ $h(s, \Lambda(s))$ and $\lambda \mapsto h(s, \lambda)$ is continuous, then $Q(s)$ is compact. Thus, $Q$ is compact valued.

Since $\Lambda$ is convex valued, then $Q$ is convex valued as well. Indeed, if $s \in S, x_{1}, x_{2} \in Q(s)$ and $a \in(0,1)$, then there exists $\lambda_{l} \in \Lambda(s)$ such that $x_{l}=h\left(s, \lambda_{l}\right)$ for all $l=1,2$. Then, $a \lambda_{1}+(1-a) \lambda_{2} \in \Lambda(s)$ and $a x_{1}+(1-a) x_{2}=$ $h\left(s, a \lambda_{1}+(1-a) \lambda_{2}\right)$ imply that $a x_{1}+(1-a) x_{2} \in Q(s)$.

Since $C \mapsto \Lambda(t, C)$ is upper hemi-continuous and $C \mapsto h(t, C)$ is continuous for all $t \in T$, then $C \mapsto Q(t, C)$ is upper hemi-continuous.

Finally, we show that $Q$ is measurable. Note first that $h$ is measurable by Lemma 4.50 in Aliprantis and Border $(1999$, p. 151) since $\Delta(\mathbb{K})$ is a compact metric space. Define $\Xi: S \rightrightarrows S \times \Delta(\mathbb{K})$ by $\Xi(s)=\{(s, \lambda): \lambda \in \Lambda(s)\}$.

We claim that $\Xi$ is measurable. Since $\Xi$ is compact valued, then it is enough to show that $\Xi$ is weakly measurable (see Aliprantis and Border (1999, Lemma 17.2, p. 559)). Let $A$ and $B$ be measurable subsets of $S$ and $\Delta(\mathbb{K})$, respectively. Then, $\Xi^{\ell}(A \times B)=\{s \in S: \Xi(s) \cap(A \times B) \neq \emptyset\}=A \cap \Lambda^{\ell}(B)$ is measurable since $\Lambda$ is measurable. Therefore, if $V=\cup_{k=1}^{\infty}\left(A_{k} \times B_{k}\right)$ and $A_{k}$ and $B_{k}$ are measurable subsets of $S$ and $\Delta(\mathbb{K})$, respectively, for all $k \in \mathbb{N}$, 
then $\Xi^{\ell}(V)=\cup_{k=1}^{\infty} \Xi^{\ell}\left(A_{k} \times B_{k}\right)$ is measurable. Therefore, if $V$ is an open subset of $S \times \Delta(\mathbb{K})$, then there exist sequences $\left\{A_{k}\right\}$ and $\left\{B_{k}\right\}$ of open subsets of $S$ and $\Delta(\mathbb{K})$ such that $V=\cup_{k=1}^{\infty}\left(A_{k} \times B_{k}\right)$ since both $S$ and $\Delta(\mathbb{K})$ are second countable. Thus, $\Xi^{\ell}(V)$ is measurable and so $\Xi$ is weakly measurable.

Since $\Xi$ is measurable, then $Q$ is measurable as well. In fact, let $B$ be a measurable subset of $\mathbb{R}^{m}$. Then, $h^{-1}(B)$ is a measurable subset of $S \times \Delta(\mathbb{K})$ and so $Q^{\ell}(B)=\left\{s \in S: \Xi(s) \cap h^{-1}(B) \neq \emptyset\right\}=\Xi^{\ell}\left(h^{-1}(B)\right)$ is measurable. This completes the proof of Lemma 1.

\section{A.3 Proof of Lemma 2}

In order to prove Lemma 2, we need the following result.

Lemma 6 Let $(S, \Sigma)$ be a measurable space, $X$ be a compact metric space and $f: S \times X \rightarrow \mathbb{R}^{m}$ be a Carathéodory function. Then, the correspondence $\zeta: S \rightrightarrows X$ defined by

$$
\zeta(s)=\{x \in X: f(s, x)=0\}
$$

is measurable.

Proof. The lemma corresponds to Aliprantis and Border (1999, Corollary 17.8 , p. 563) when $m=1$. To extend it to an arbitrary $m \in \mathbb{N}$, simply substitute the absolute value (in $\mathbb{R}$ ) used in the proof of that corollary by a norm in $\mathbb{R}^{m}$.

We next turn to the proof of Lemma 2. Consider $H: S \rightrightarrows X$ defined by

$$
H(s)=\{x \in X: g(s, x)=q(s)\} .
$$


If there exists a measurable selection $\sigma$ from $H \cap \Theta$, then $q(s)=g(s, \sigma(s))$ for all $s \in S$. So, it is enough to show that $H \cap \Theta$ has a measurable selection.

Note that $H \cap \Theta$ is nonempty valued. This is so because $q(s) \in Q(s)=$ $\{g(s, x): x \in \Theta(s)\}$. Since $x \mapsto g(s, x)$ is continuous for all $s \in S$, then $H(s)$ is a closed subset of $X$, hence compact. Thus, $H$ is compact valued, and so is $H \cap \Theta$.

Let $f: S \times X \rightarrow \mathbb{R}^{m}$ be defined by

$$
f(s, x)=g(s, x)-q(s)
$$

Then, $f$ is measurable in $s$ and continuous in $x$. Note that $H(s)=\{x \in$ $X: f(s, x)=0\}$ for all $s \in S$. Since $S$ is a measurable space (with its Borel $\sigma$-algebra) and $X$ is a compact metric space, it follows by Lemma 6 that $H$ is measurable. Then, $H \cap \Theta$ is measurable by Aliprantis and Border (1999, Lemma 17.4 .3 , p. 560). Then, $H \cap \Theta$ is weakly measurable (Aliprantis and Border (1999, Lemma 17.2.1, p. 559)) and so by the Kuratowski-RyllNardzewski Theorem (see Aliprantis and Border (1999, Theorem 17.13, p. 567)) $H \cap \Theta$ has a measurable selection.

\section{A.4 Properties of $\varphi$}

In this appendix, we establish the properties of the agent's constraint correspondences $\varphi^{P M}, \varphi^{M S}$ and $\varphi^{H}$. In all these cases, the result is a consequence of the following Lemma.

Lemma 7 If $\phi: P \rightrightarrows \mathbb{K}$ is continuous with nonempty compact values, then $\varphi: T \times P \rightrightarrows \Delta(\mathbb{K})$ defined by

$$
\varphi(t, C)=\{\lambda \in \Delta(\mathbb{K}): \lambda(\phi(C))=1\}
$$


is continuous and has nonempty, convex, compact values.

Proof. It follows from Aliprantis and Border (1999, Theorem 16.14, p. 530) that $\varphi$ is upper hemi-continuous with nonempty, compact, convex values.

We claim that $\varphi$ is also lower hemi-continuous. In order to prove this claim, let $\left\{t_{n}\right\}_{n=1}^{\infty}$ be a convergent sequence in $T,\left\{C_{n}\right\}_{n=1}^{\infty}$ be a convergent sequence in $P, t=\lim _{n} t_{n}, C=\lim _{n} C_{n}$ and $\lambda \in \varphi(t, C)$. We need to prove that there exists a subsequence $\left\{n_{j}\right\}_{j=1}^{\infty}$ of indexes and elements $\lambda_{n_{j}} \in$ $\varphi\left(t_{n_{j}}, C_{n_{j}}\right)$ such that $\lambda_{n_{j}}$ converges to $\lambda$.

By Aliprantis and Border (1999, Theorem 16.16, p. 531), the function $\Phi$ from $P$ into the space of all nonempty, compact subsets of $\mathbb{K}$ endowed with the Hausdorff metric defined by $\Phi(C)=\phi(C)$ is continuous. Thus, letting $d_{H}$ denote the Hausdorff metric, it follows that $d_{H}\left(\phi\left(C_{n}\right), \phi(C)\right)$ converges to zero.

For all $j \in \mathbb{N}$, let $n_{j} \in \mathbb{N}$ be such that $d_{H}\left(\phi\left(C_{n_{j}}\right), \phi(C)\right)<1 / j$. Let $j$ be fixed. Since $d_{H}\left(\phi\left(C_{n_{j}}\right), \phi(C)\right)<1 / j$, then $\phi(C) \subseteq \cup_{k \in \phi\left(C_{n_{j}}\right)} B_{1 / j}(k)$. Since $\phi(C)$ is a compact subset of $\mathbb{K}$, then there exists $\left\{k_{1}, \ldots, k_{M}\right\} \subseteq \phi\left(C_{n_{j}}\right)$ such that $\phi(C) \subseteq \cup_{m=1}^{M} B_{1 / j}\left(k_{m}\right)$.

Finally, define $B_{1}=B_{1 / j}\left(k_{1}\right), B_{m}=B_{1 / j}\left(k_{m}\right) \backslash \cup_{l=1}^{m-1} B_{l}$ for all $m=$ $2, \ldots, M$, and $\lambda_{n_{j}}$ by setting $\lambda_{n_{j}}\left(k_{m}\right)=\lambda\left(B_{m}\right)$ for all $m=1, \ldots, M$.

Since $\lambda(\phi(C))=1$, then $\lambda_{n_{j}}\left(\phi\left(C_{n_{j}}\right)\right)=1$ and, arguing as in the proof of Parthasarathy (1967, Theorem II.6.3), it follows that $\lambda_{n_{j}}$ converges to $\lambda$. Therefore, $\varphi$ is lower hemi-continuous.

As a consequence, we obtain the following corollary. 
Corollary 2 The correspondences $\varphi^{P M}, \varphi^{M S}$ and $\varphi^{H}$ are continuous with nonempty, convex, compact values.

Proof. In the case of $\varphi^{M S}$, simply define $\phi^{M S}: P \rightrightarrows \mathbb{K}^{M S}$ by $\phi^{M S}(C)=$ $C$. Clearly, $\phi^{M S}$ is continuous and has nonempty compact values.

In the case $\varphi^{P M}$, we define for all $i \in I, \phi_{i}: P \rightrightarrows \mathbb{K}^{P M}$ by $\phi_{i}(C)=\{i\} \times C_{i}$ and $\phi^{P M}: P \rightrightarrows \mathbb{K}^{P M}$ by $\phi^{P M}(C)=\cup_{i=1}^{m} \phi_{i}(C)$. Clearly, $\phi^{P M}$ has nonempty and compact values and $\phi_{i}$ is continuous for all $i \in I$. Since the finite union of continuous correspondences is continuous (see Aliprantis and Border (1999, Theorem 16.27, p. 537)), then $\phi^{P M}$ is continuous.

In the hybrid case, we define for $j=1,2, \phi_{j}: P \rightrightarrows \mathbb{K}^{H}$, by $\phi_{1}(C)=$ $\cup_{i \in I_{e}}\{i\} \times C_{i}$ and $\phi_{2}(C)=\prod_{i \in I_{e}^{c}} C_{i}$. These correspondences are continuous and have nonempty and compact values. Finally, define $\phi^{H}: P \rightrightarrows \mathbb{K}^{H}$ by $\phi^{H}(C)=\phi_{1}(C) \times \phi_{2}(C)$. Since the finite product of continuous correspondences with compact values is continuous (see Aliprantis and Border (1999, Theorem 16.28, p. 537)), then $\phi^{H}$ is continuous and has nonempty and compact values as well.

In all cases the conclusion follows from Lemma 7.

\section{References}

Aliprantis, C., and K. Border (1999): Infinite Dimensional Analysis. Springer, Berlin.

Berge, C. (1997): Topological Spaces. Dover, New York. 
Carmona, G., and J. Fajardo (2006): "On the Definition of Equilibrium in Common Agency Games with Adverse Selection," mimeo, Universidade Nova de Lisboa and IBMEC Business School.

Hildenbrand, W. (1974): Core and Equilibria of a Large Economy. Princeton University Press, Princeton.

Kreps, D. M., And R. Wilson (1982): "Sequential Equilibria," Econometrica, 50(4), 863-894.

Martimort, D. (2006): "Multi-Contracting Mechanism Design," mimeo, IDEI Toulouse.

Martimort, D., And H. Moreira (2006): "Common Agency with Informed Principals," mimeo, IDEI Toulouse.

Martimort, D., and L. Stole (2002): "The Revelation and Delegation Principles in Common Agency Games," Econometrica, 70(4), 1659-1673.

Maskin, E., And J. Tirole (1990): "The Principal-Agent Relationship with an Informed Principal: The Case of Private Values," Econometrica, 58(2), 379-409.

Monteiro, P., and F. Page (2005): "Existence of Nash Equilibrium in Competitive Nonlinear Pricing Games with Adverse Selection," mimeo, Fundação Getúlio Vargas and University of Alabama.

Page, F., And P. Monteiro (2003): "Three Principles of Competitive Nonlinear Pricing," Journal of Mathematical Economics, 39, 63-109. 
Parthasarathy, K. (1967): Probability Measures on Metric Spaces. Academic Press, New York.

Peters, M. (2001): "Common Agency and the Revelation Principle," Econometrica, 69(5), 1349-1372.

(2003): "Negotiation and Take-It-Or-Leave-It in Common Agency," Journal of Economic Theory, 111(1), 88-109.

Simon, L., And W. Zame (1990): "Discontinuous Games and Endogenous Sharing Rules," Econometrica, 58(4), 861-872. 